\title{
Effect of Si Substitution and Annealing on Magnetocaloric Properties in $\mathrm{TbCo}_{2}$
}

\author{
M. Janatova, J. Poltierova Vejpravova, P. Javorsky, \\ J. Prokleska, P. Svoboda And S. Danis
}

Charles University Prague, Faculty of Mathematics and Physics, DCMP

Ke Karlovu 5, 12116 Prague 2, Czech Republic

\begin{abstract}
We report on magnetocaloric properties of the as-cast and annealed $\mathrm{TbCO}_{2}$ with partial substitution of cobalt by silicon. The samples were characterized by powder X-ray diffraction and investigated by heat capacity measurements $(8-300 \mathrm{~K}$, in fields $0 \mathrm{~T}$ and $1 \mathrm{~T})$. $\mathrm{TbCo}_{2}$ undergoes a second-order type phase transition at $T_{\mathrm{C}}=240 \mathrm{~K}$, from paramagnetic to ferrimagnetic state; similar behavior was revealed in the Si-doped compounds. The temperature dependence of magnetic entropy and the corresponding magnetocaloric effect were derived for all studied samples. The influence of the heat treatment and $\mathrm{Si}$ doping on magnetocaloric properties will be discussed in context of the analogue case $\operatorname{Er}\left(\mathrm{Co}_{1-x} \mathrm{Si}_{x}\right)_{2}$.
\end{abstract}

PACS numbers: 65.40.Gd, 75.30.Sg

\section{Introduction}

A phenomenon of magnetocaloric effect (MCE), which is detected as a sample temperature change due to application of a magnetic field to a magnetic material, was originally observed on iron by Emil Warburg [1]. MCE can be quantified as the isothermal magnetic entropy change $\Delta S$ or the adiabatic temperature change $\Delta T_{\text {ad }}$.

Recently, increased interest in the MCE is due to application of magnetic materials with giant MCE as refrigerators for environmentally-friendly magnetic cooling [2]. At present, rare-earth (RE) based intermetallic $\mathrm{RECo}_{2}$ compounds are subject of intensive studies because of their sensitivity to external magnetic field change, which means a large magnetic entropy change in the vicinity of the magnetic phase transition leading to large MCE. Moreover, partial substitution of cobalt by another element (e.g. by silicon or germanium) can dramatically modify the magnetic properties of the compound. For example in $\operatorname{Er}\left(\mathrm{Co}_{1-x} \mathrm{Si}_{x}\right)_{2}$ system, a partial substitution of cobalt by silicon caused a shift of the Curie temperature, $T_{\mathrm{C}}=53 \mathrm{~K}$ in $\operatorname{Er}\left(\mathrm{Co}_{0.95} \mathrm{Si}_{0.05}\right)_{2}$ in contrast to the $\mathrm{ErCo}_{2}$ with $T_{\mathrm{C}}=33 \mathrm{~K}$ [3]. 
In our paper, we are focusing on $\mathrm{TbCo}_{2}$ compound, which is ferrimagnetic below $T_{\mathrm{C}}=240 \mathrm{~K}$ and exhibits the second order transition at $T_{\mathrm{C}}$ [4].

The goal of this paper is to investigate MCE in the vicinity of the magnetic phase transition in $\mathrm{TbCo}_{1.98} \mathrm{Si}_{0.02}$ compound and to compare the results of our measurements with results for $\mathrm{TbCo}_{2}$ compound without substitution. Moreover, effect of additional heat treatment on the value of MCE will be discussed.

\section{Experimental}

Two polycrystalline samples of $\mathrm{TbCo}_{1.98} \mathrm{Si}_{0.02}$ were synthesized by arc melting of constituent elements $\mathrm{Tb}(3 \mathrm{~N}), \mathrm{Co}(3 \mathrm{~N})$ and $\mathrm{Si}(5 \mathrm{~N})$ in a protective argon atmosphere in a water-cooled copper crucible. The samples were several times re-melted to improve homogeneity. The second sample was annealed at $950^{\circ} \mathrm{C}$ for $50 \mathrm{~h}$. As-cast and annealed samples were characterized by powder X-ray diffraction (XRD). The heat capacity measurements were performed using the PPMS (Quantum Design) facility in fields $0 \mathrm{~T}$ and $1 \mathrm{~T}$ in temperature range 8-300 K.

\section{Results and discussion}

The XRD-pattern analysis revealed a contamination by $\mathrm{TbCo}_{3}$ in the as-cast sample, while the annealed sample was single-phase.

Temperature dependences of the specific heat are presented in Fig. 1.
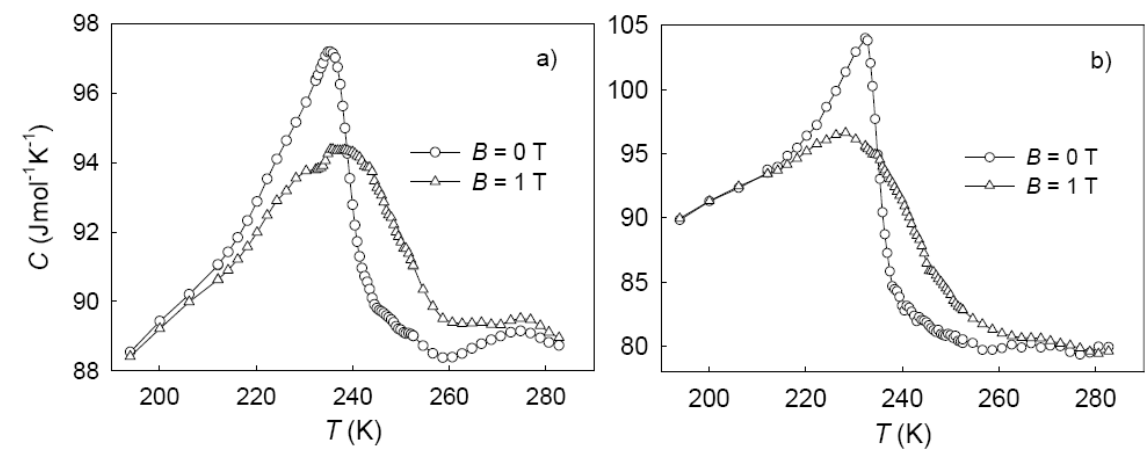

Fig. 1. Temperature dependence of the specific heat near the magnetic phase transition for the as-cast (a) and annealed (b) sample, respectively.

The temperature dependence of the total entropy was calculated from the measured specific heat data. Then the MCE was determined as the isothermal entropy change, $-\Delta S$ and the adiabatic temperature change, $\Delta T_{\mathrm{ad}}$.

The partial substitution of cobalt by silicon caused a decrease in the Curie temperature, $T_{\mathrm{C}}$ to $235 \mathrm{~K}$ in the annealed $\mathrm{TbCo}_{1.98} \mathrm{Si}_{0.02}$ in comparison to the $T_{\mathrm{C}}=240 \mathrm{~K}$ in $\mathrm{TbCo}_{2}$.

The obtained results of $-\Delta S$ and $\Delta T_{\mathrm{ad}}$ for both samples are presented in Fig. 2 . 

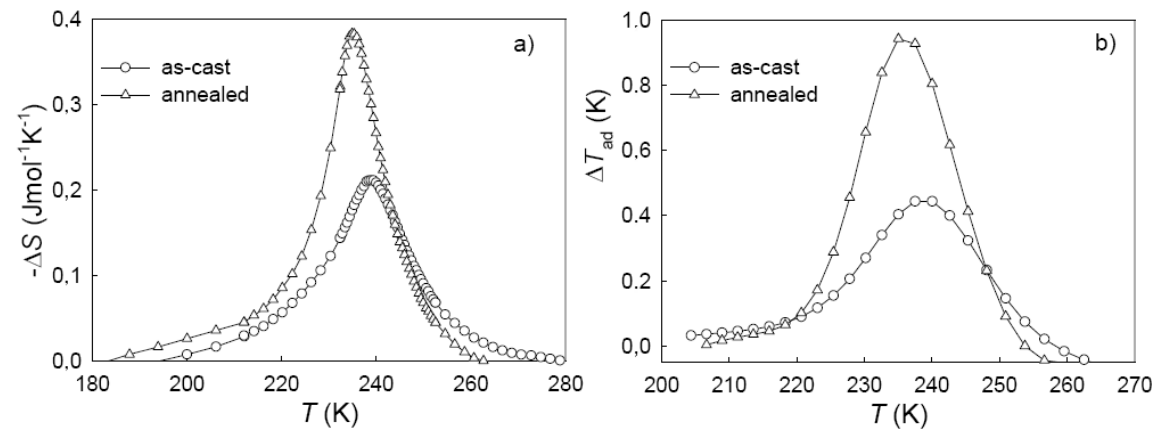

Fig. 2. The entropy change (a) and the adiabatic temperature change (b) of the as-cast and annealed sample in $\Delta B=1 \mathrm{~T}$.

It is evident that temperature dependence of the isothermal entropy change, respectively the adiabatic temperature change has the maximum $-\Delta S^{\max }$ and $\Delta T_{\mathrm{ad}}^{\max }$ at $\approx T_{\mathrm{C}}$, respectively.

The resulting values of both quantities are: for the as-cast $\mathrm{TbCo}_{1.98} \mathrm{Si}_{0.02}$, $\left(-\Delta S^{\max }\right)=0.21 \mathrm{~J} \mathrm{~mol}^{-1} \mathrm{~K}^{-1}$ and $\Delta T_{\mathrm{ad}}^{\max }=0.44 \mathrm{~K}$ in the magnetic field change, $\Delta B=1 \mathrm{~T}$, and for the annealed $\mathrm{TbCo}_{1.98} \mathrm{Si}_{0.02}$ the $\left(-\Delta S^{\max }\right)$ reaches a value of $0.38 \mathrm{~J} \mathrm{~mol}^{-1} \mathrm{~K}^{-1}$ and $\Delta T_{\mathrm{ad}}^{\max }=0.94 \mathrm{~K}$ in the same magnetic field change, respectively. For comparison, the value of the isothermal entropy change for the pure $\mathrm{TbCo}_{2}$ without substitution is $\left(-\Delta S^{\max }\right)=0.9 \mathrm{~J} \mathrm{~mol}^{-1} \mathrm{~K}^{-1}[5]$.

The observed behavior is evidently in contrast to the effect of Si substitution in the isostructural $\mathrm{ErCo}_{2}$ case; the reason might be the nature of the magnetic phase transition at $T_{\mathrm{C}}$ of the rare-earth sublattices. The phenomena accompanying the magnetic ordering transition in $\mathrm{ErCO}_{2}$ are intimately connected with the magnetic instability in the Co sublattice at $T_{\mathrm{C}}$. This is associated with the formation of the $3 d$-electron magnetic moment due to the itinerant $3 d$-electron metamagnetism induced by the molecular field from the $4 f$-electron moments in the $\mathrm{RE}$ sublattice, which order ferromagnetically at $T_{\mathrm{C}}$. The magnetic phase transition in $\mathrm{ErCo}_{2}$ at $T_{\mathrm{C}}$ is therefore of the first-order.

In addition, above $T_{\mathrm{C}}$ a metamagnetic transition takes place when applying a magnetic field, which is accompanied by a magnetic entropy change. This is the origin of the large MCE.

We may speculate that the enhancement of the spin fluctuations by $\mathrm{Si}$ substitution is the dominant mechanism leading to the increase in the $T_{\mathrm{C}}$ in $\operatorname{Er}\left(\mathrm{Co}_{1-x} \mathrm{Si}_{x}\right)_{2}$ compounds. In $\mathrm{TbCo}_{2}$, the $T_{\mathrm{C}}$-related transition is of the second order lacking the phenomena (metamagnetism above $T_{\mathrm{C}}$ etc.) observed in $\mathrm{ErCo}_{2}$ governed by the strong spin fluctuations on the Co sublattice. Therefore, common effect of substitution by means of the structural disorder and dilution of the exchange interaction is observed. 


\section{Conclusions}

The magnetocaloric effect of the as-cast and annealed $\mathrm{TbCo}_{1.98} \mathrm{Si}_{0.02}$ compound was studied by the specific heat measurements. Annealing procedure has a positive influence on the value of MCE, because the presence of another phase in the as-cast sample reduced the absolute value of the MCE. There is also a considerable reduction of the value of $\mathrm{MCE}$ in $\mathrm{TbCo}_{2}$ derivative in comparison to the pure $\mathrm{TbCo}_{2}$. Moreover, in contrast to the $\mathrm{Si}$ doped $\mathrm{ErCo}_{2}$, the $T_{\mathrm{C}}$ is shifted to lower temperatures, and the $T_{\mathrm{C}}$-related anomaly on the specific heat is much broadened by the common disorder effects induced by the Si substitution.

\section{Acknowledgments}

This work is a part of the research plan MSM 0021620834 financed by the Ministry of Education of the Czech Republic. It was also supported by the Grant Agency of the Charles University (project No. 209/2006).

\section{References}

[1] E. Warburg, Ann. Phys. (Leipzig), 13, 1441 (1881).

[2] V.K. Pecharsky, K.A. Gschneider Jr., J. Magn. Magn. Mater. 200, 44 (1999).

[3] D. Vasylyev, J. Prokleska, J. Sebek, V. Sechovsky, J. Alloys Comp. 394, 96 (2005).

[4] D. Gignoux, F. Givord, F. Sayetat, J. Magn. Magn. Mater. 15-18, 1235 (1980).

[5] N.H. Duc, D.T. Kim Anh, J. Magn. Magn. Mater. 242-245, 873 (2002). 